\title{
Comparison of the GUM and Monte Carlo methods on the flatness uncertainty estimation in coordinate measuring machine
}

\author{
Abdelilah Jalid $^{1 \star}$, Said Hariri² ${ }^{2}$ Abdellah El Gharad ${ }^{1}$, and Jean Paul Senelaer ${ }^{3}$ \\ 1 Univesité Mohammed V de Rabat, École Normale Supérieure d'Enseignement Technique (ENSET ) de Rabat, \\ Laboratoire LaMIPI, Avenue de l'Armée Royale, Madinat Al Irfane, 10100 Rabat, Maroc \\ 2 Département Technologie des Polymères et Composites et Ingénierie Mécanique Ecole des Mines de Douai, \\ 941 rue Charles Bourseul, 59500 Douai, France \\ 3 École Supérieure de Métrologie, École des Mines de Douai, 59500 Douai, France
}

Received: 16 November 2015 / Accepted: 3rd May 2016

\begin{abstract}
In engineering industry, control of manufactured parts is usually done on a coordinate measuring machine (CMM), a sensor mounted at the end of the machine probes a set of points on the surface to be inspected. Data processing is performed subsequently using software, and the result of this measurement process either validates or not the conformity of the part. Measurement uncertainty is a crucial parameter for making the right decisions, and not taking into account this parameter can, therefore, sometimes lead to aberrant decisions. The determination of the uncertainty measurement on CMM is a complex task for the variety of influencing factors. Through this study, we aim to check if the uncertainty propagation model developed according to the guide to the expression of uncertainty in measurement (GUM) approach is valid, we present here a comparison of the GUM and Monte Carlo methods. This comparison is made to estimate a flatness deviation of a surface belonging to an industrial part and the uncertainty associated to the measurement result.
\end{abstract}

Keywords: CMM uncertainty, flatness uncertainty, GUM uncertainty, Monte Carlo method

\section{Introduction}

In mechanical industry, control of manufactured parts is usually done by a coordinate measuring machine (CMM), with a sensor mounted at the end of the machine. A set of data points are palpated on the surface to be inspected; software processing of the data sets to estimate the flatness default of this element. The result of this measurement process either validates or not the conformity of the part. Measurement uncertainty is a critical parameter to make the right decisions. Therefore, not taking into account this setting can sometimes lead to aberrant decisions. The estimation of measurement uncertainty is essential for high quality part production and the determination of this parameter in a CMM is a complex task, given the diversity of influences. Many studies have focused on the estimation of uncertainty associated with the measurement result. Sladek presents in reference [1] a method of estimating the measurement uncertainty by Monte Carlo method based on the model of the virtual machine. Wen proposes in reference [2] an evaluation process and verification of flatness related to the GPS standard, taking into account

* Correspondence: jalid.abdel@gmail.com the associated uncertainty. Flatness error is calculated using a mathematical model based on a genetic algorithm. Forbes presents in reference [3] the various approaches for estimating measurement uncertainties, and demonstrates that the GUM method and Monte Carlo method provide approximate solutions to Bayesian method. Balsamo et al. [4] evaluates the uncertainty of CMM through Monte Carlo simulations. Kruth [5] presents a method for estimating the uncertainty of measurement on a CMM and the Monte Carlo simulation shows that the sample size taken from a surface is a factor that influences the measurement uncertainty. Cui et al. [6], present the evaluation datum by using both the least squares method and the genetic optimization algorithm. Their computation uncertainties to flatness and roundness were compared with each other using the sample data from a coordinate measurement. Cox et al. [7], define the GUM as an approximative method for the evaluation of uncertainty and explain that a Monte Carlo method is an effective and versatile tool for determining the PDF for the measurands. This method provides a consistent Bayesian approach to the evaluation of uncertainty. Yang et al. [8], estimate the uncertainty of task-specific laser tracker measurements by using the GUM and the Monte Carlo method, a case 

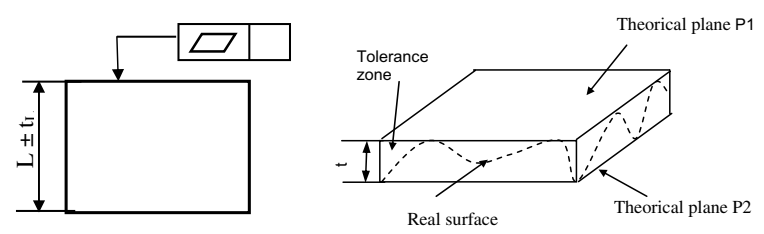

Fig. 1. Flatness deviation.

study involving the uncertainty estimation of a cylindricity measurement process was illustrated. The results indicate that the Monte Carlo method is a practical tool for applying the principle of propagation of distributions and does not depend on the assumptions and limitations required by the law of propagation of uncertainties. Diaz et al. [9], have shown that a data processing algorithm can contribute significantly to the total measurement error of a CMM.

All the methods proposed, treat the estimation of measurement uncertainty of CMM, these studies assume that the coordinates of the measured points are not affected with error, which is not true on a CMM. They also estimate the uncertainty measurement by one or the other method.

In this paper, we present a comparative study between two methods for estimating the measurement uncertainty associated to the flatness error. The first is based on the GUM approach defined in the guideline [10], a model is developed according to this approach, which takes into account the uncertainty coordinate's data points, while the second is a numerical method of Monte Carlo (MCM) defined in Supplement 1 of the GUM [11].

In this article, we have also presented a method to calculate how the uncertainty coordinate's data points are propagated through to uncertainties associated with the parameters describing the fitted surface.

\section{Mathematical modeling of the flatness of a surface}

Flatness is defined in ISO 1101 [12], as the minimum distance, between two parallel planes P1 and P2 containing all sampled points (see Fig. 1).

From data sets of points, taken on a mechanical part in CMM, the software associated with the machine, assesses a substitute feature of the measured surface by applying the least squares or minimax criteria; the parameters of the associated feature are used in a calculation step, to estimate the flatness error.

$\vec{n}$ represents the direction of the normal vector, to the substitute feature (plane in our case), $M$ and $m$ are the two extreme points of the measured points in this direction. This is shown in Figure 2.

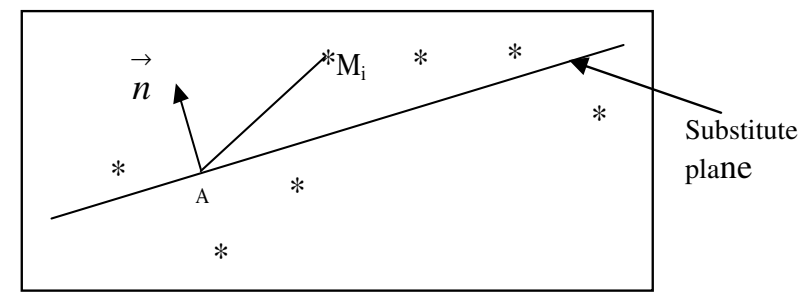

Fig. 2. Parameters of the substitute plane.

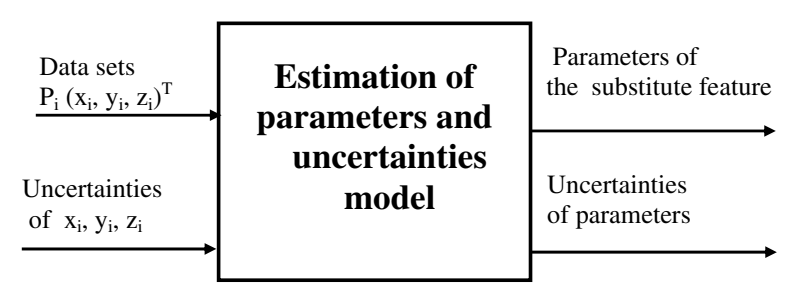

Fig. 3. Input and outputs of the estimation parameters and uncertainties model.

The flatness error, can be estimated from the expression:

$$
\begin{aligned}
d p & =|\overrightarrow{M m} \cdot \vec{n}| \\
d p & =\left|\left(\begin{array}{l}
x_{m}-x_{M} \\
y_{m}-y_{M} \\
z_{m}-z_{M}
\end{array}\right)\left(\begin{array}{c}
n_{x} \\
n_{y} \\
n_{z}
\end{array}\right)\right| \\
& =\left|\left(x_{m}-x_{M}\right) n_{x}+\left(y_{m}-y_{M}\right) n_{y}+\left(z_{m}-z_{M}\right) n_{z}\right| .
\end{aligned}
$$

The parameters estimation of the substitute plan, is made according to the model shown in Figure 3; the model is based on the orthogonal distance regression (ODR) [13] and provides, the parameter of the substitute feature and the associated uncertainties. This estimation, takes into account the uncertainties of the coordinate's data points. A method is developed in reference [14], which gives more details about the algorithm and the adopted approach. We have given in the Appendix an overview of the method used (Fig. 3).

\subsection{Standard uncertainty on the coordinates of points}

The uncertainty of a point acquisition is not generally constant and depends on the position of the measured point. The evaluation of this uncertainty requires repeatability and reproducibility tests which are complicated procedures and demand a good knowledge of the machine. Some simplifications can be made initially; one of the relations commonly used is illustrated by the following form [15]:

$$
u=\frac{\sqrt{\left(a^{2}+(b \cdot L)^{2}\right.}}{k}
$$

where " $a$ " and " $b$ " are constants, and " $k$ " the coverage factor defined by the law of the selected distribution. 


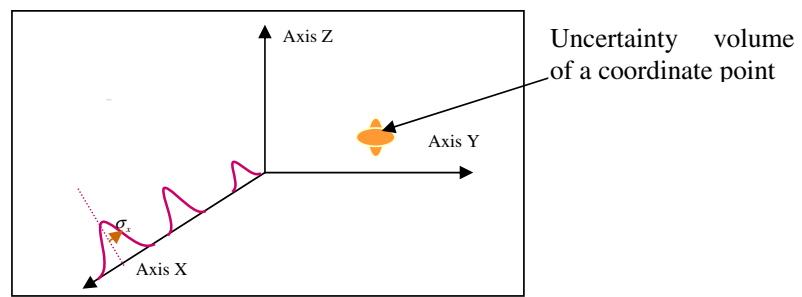

Fig. 4. Uncertainty volume of a coordinates point.

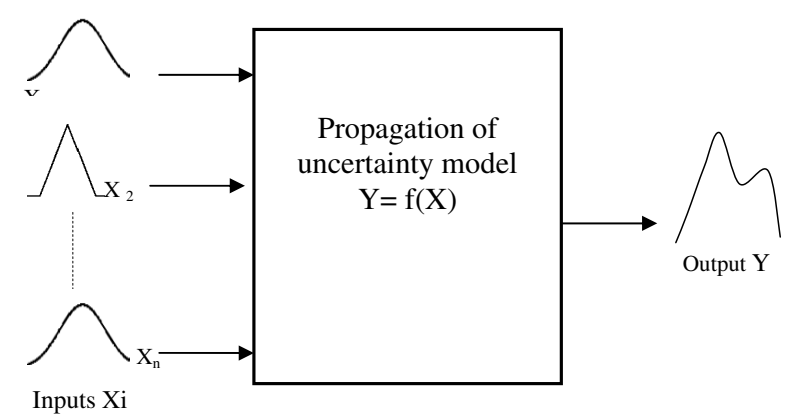

Fig. 5. Propagation of uncertainty model.

This standard uncertainty is defined for each axis of the machine. By making the assumption that the distribution of these errors follows a normal law with null average and $\sigma=u$ as the standard deviation, one will have $\sigma_{x}$ as the standard deviation of the distribution along axis $x, \sigma_{y}$ for axis $y$ and $\sigma_{z}$ for axis $z$. This is shown in Figure 4 .

The weighting is introduced to compensate for the case where the measuring accuracy is not the same for all points. This is especially the case in a CMM, since the uncertainty in the coordinates depends on the value read on the rules of the machine.

For each measured point of coordinate $\left(x_{i}, y_{i}, z_{i}\right)^{T}$ one applies a weighting $\omega_{i}$. This weighting is in the form $\omega_{i}=$ $\frac{\alpha}{\sigma_{i}^{2}}$. The number $\alpha$ is used to represent the importance of a measuring point in relation to another. In this case one will take $\alpha=1$ which means that all the measured points have an equal importance.

For each observation $i$, there will be a matrix of order weighting $(3,3)$ given as follows:

$$
\left[\begin{array}{ccc}
\frac{1}{\sigma_{x_{i}}^{2}} & 0 & 0 \\
0 & \frac{1}{\sigma_{y_{i}}^{2}} & 0 \\
0 & 0 & \frac{1}{\sigma_{z_{i}}^{2}}
\end{array}\right] .
$$

\section{Propagation of uncertainty models}

$Y=f\left(X_{i}\right)$ is a function of several variables, and $X_{i}$ is defined as random variables with $\hat{X}$ as a mean value and $\sigma_{x}$ as standard deviation. The output $Y$ has $\hat{Y}$ as a mean value and $\sigma_{y}$ as standard deviation. The determination of uncertainty propagation mechanism is to seek the estimator $\hat{Y}$ and the variance $V(Y)$; this can be illustrated by Figure 5.
Among the different approaches for the mechanism of uncertainty propagation, we can cite.

- The law of propagation of uncertainty GUM.

- The Monte Carlo method.

\subsection{The law of propagation of uncertainty GUM}

This law is based on a Taylor expansion of the function $Y=f(X)$ in the first order. The variance of the output $Y$ is expressed by the following formula:

$$
\begin{aligned}
V(Y)= & \sum_{i=1}^{k}\left[\left(\frac{\partial f}{\partial X_{i}}\right)_{X=\mu} V\left(X_{i}\right)\right]^{2} \\
& +2 \sum_{i=1}^{k-1} \sum_{j>i}^{k}\left(\frac{\partial f}{\partial X_{i}}\right)_{X=\mu}\left(\frac{\partial f}{\partial X_{j}}\right)_{X=\mu} \\
& \times \operatorname{cov}\left(X_{i}, X_{j}\right) .
\end{aligned}
$$

This law is defined in the Guide to the Expression of Uncertainty in Measurement [10]. It allows us to estimate the variance of the output $Y$ from the knowledge of variance of the input variables $X_{i}$.

\subsubsection{Generalized model propagation}

This is the generalization of the equation (5) for several output variables $Y$. For a vector function $Y=$ $f\left(X_{1}, X_{2}, X_{3}, \ldots, X_{n}\right)$, we have the following:

$$
\begin{aligned}
M V C(Y)= & \sum_{i=1}^{k}\left[\left(\frac{\partial f}{\partial X_{i}}\right)_{X=\mu} V\left(X_{i}\right)\right]^{2} \\
& +2 \sum_{i=1}^{k-1} \sum_{j>i}^{k}\left(\frac{\partial f}{\partial X_{i}}\right)_{X=\mu}\left(\frac{\partial f}{\partial X_{j}}\right)_{X=\mu} \\
& \times \operatorname{cov}\left(X_{i}, X_{j}\right) \\
= & \sum_{i=1}^{k} \sum_{j=1}^{k}\left(\frac{\partial f}{\partial X_{i}}\right)_{X=\mu}\left(\frac{\partial f}{\partial X_{j}}\right)_{X=\mu} \operatorname{cov}\left(X_{i}, X_{j}\right) \\
= & J \Omega J^{T}
\end{aligned}
$$

where

$-\mu=\hat{X}$ represents the estimator of vector $\hat{X}\left(\hat{X}_{1}, \hat{X}_{2}, \ldots, \hat{X}_{n}\right)$

- $J$ is the Jacobian matrix,

- $M V C(Y)$ represents the variance-covariance matrix of vector $Y$,

- $\Omega$ represents the matrix of variance covariance of parameters $n \rightarrow, m$, and $M$. This matrix is given below:

$$
\Omega=\left[\begin{array}{lll}
{\left[n^{\rightarrow}\right]} & & \\
& {[m]} & \\
& & \\
& & {[M]}
\end{array}\right]
$$




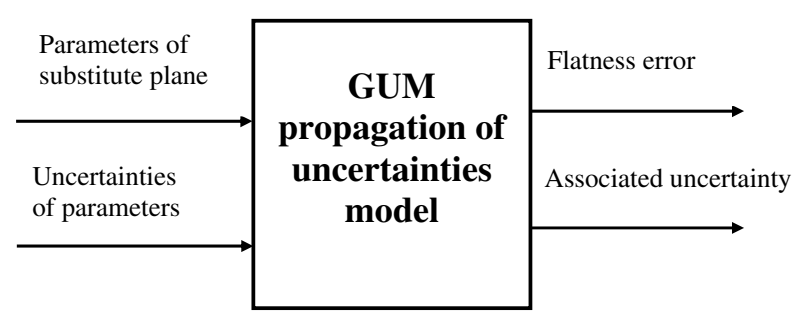

Fig. 6. Input and outputs of the GUM propagation of uncertainties model.

and

$$
\left[n^{\rightarrow}\right]=\left(\begin{array}{ccc}
\operatorname{var}\left(n_{x}\right) & \operatorname{cov}\left(n_{x}, n_{y}\right) & \operatorname{cov}\left(n_{x}, n_{z}\right) \\
\operatorname{cov}\left(n_{y}, n_{x}\right) & \operatorname{var}\left(n_{y}\right) & \operatorname{cov}\left(n_{y}, n_{z}\right) \\
\operatorname{cov}\left(n_{z}, n_{x}\right) & \operatorname{cov}\left(n_{z}, n_{y}\right) & \operatorname{var}\left(n_{z}\right)
\end{array}\right)
$$

This general law of uncertainty propagation, can be written in the following condensed form:

$$
M V C(Y)=J \Omega J^{T} .
$$

This formulation, will be subsequently adopted to express the uncertainty propagation, from the parameters of the substitute feature to the flatness error. The model based on this formulation is described in detail in references $[14,16]$. The inputs and outputs of model are shown in Figure 6.

\subsection{Monte Carlo method}

The Monte Carlo method, is a general method, used to estimate the uncertainty propagation. This approach, can be used in many situations, where the GUM uncertainty framework is difficult or infeasible to apply, such as those in which the models are very complicated. The partial derivatives of the model are difficult to obtain.

The MCM reconstitute artificially a random phenomenon, by simulating a fictitious sample given from the input variables. We must, therefore, define the probability densities, of each input variable to be propagated, to obtain the probability density function of the output variable.

This method, can serve as a validation approach, for the law of propagation of uncertainty, by making a comparison of results.

\subsection{Validation of the GUM approach by Monte Carlo simulation}

The proposed approach by the Supplement 1 of the GUM [11] on numerical methods has the following objective: determining whether the coverage intervals obtained by the GUM uncertainty framework and MCM are in agreement with a numerical tolerance. This document,

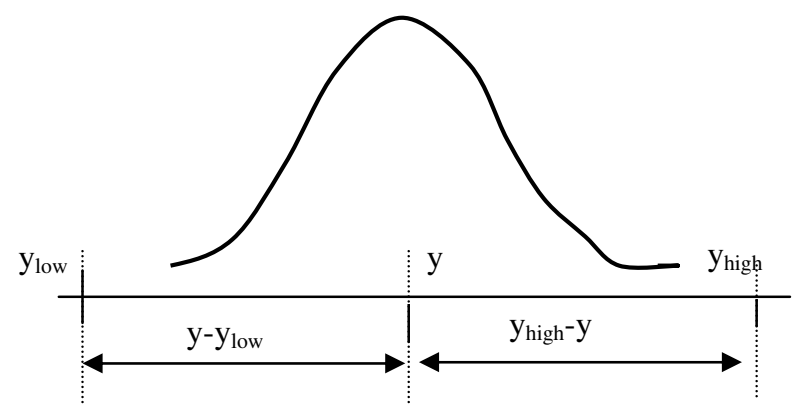

Fig. 7. Monte Carlo distribution parameters.

describes a procedure to valid the GUM as follows:

- Apply the GUM uncertainty framework; the result of GUM propagation uncertainty is in the form $y \pm U(y)$.

- Apply the adaptive Monte Carlo procedure; the Monte Carlo simulation generates a distribution with $y$ as the mean value and $u(y)$ as standard deviation.

For comparison:

- Form a numerical tolerance $\xi$ associated with $u(y)$. We express the degree of approximation in the form:

$$
\xi=(1 / 2) 10^{r}
$$

- Compare the coverage intervals obtained by the GUM uncertainty framework and MCM to determine, whether the required number of correct decimal digits in the coverage interval provided by the GUM uncertainty framework has been obtained. Specifically, determine the following quantities:

$$
\begin{aligned}
& -d_{\text {low }}=\left|y-U(y)-y_{\text {low }}\right|, \\
& -d_{\text {high }}=\left|y+U(y)-y_{\text {high }}\right|,
\end{aligned}
$$

where $y_{\text {low }}$ and $y_{\text {high }}$ are two limits of the Monte Carlo distribution calculated for a 95\% confidence interval. Then if both dlow and dhigh, are no larger than $\xi$, the comparison is favourable and the GUM uncertainty framework, has been validated in this instance (see Fig. 7).

This validation process will be used to verify in Section 4.3 the developed algorithm based on the GUM uncertainty.

\section{Application in dimensional metrology}

The surface of an industrial part was measured in 24 points. The surface to be controlled is placed on the table of the machine with an approximate direction carried by the vertical axis $z$ of the machine. The data sets points are given in Table 1.

By applying the least squares criterion, the estimate of the substitute plan gives us the results in Table 2.

Where $A\left(x_{A}, y_{A}, z_{A}\right)^{t}$ is a point of the substitute feature and $\vec{n}\left(n_{x}, n_{y}, n_{z}\right)^{t}$ the normal vector to this surface. 
Table 1. Data points.

\begin{tabular}{ccccccc}
\hline$x$ & $y$ & $z$ & & $x$ & $y$ & $z$ \\
\hline 24.921 & -29.972 & -0.002 & & 25.006 & -10.003 & -0.008 \\
-20.204 & -30.002 & -0.004 & & -24.764 & 0.008 & -0.006 \\
-15.013 & -30.011 & -0.004 & & -20.111 & 0.002 & -0.005 \\
-9.999 & -30.018 & -0.004 & & 20.000 & -0.004 & -0.009 \\
-4.962 & -30.027 & -0.006 & & -9.985 & 30.001 & -0.006 \\
-0.025 & -30.034 & -0.005 & & -4.984 & 30.000 & -0.007 \\
4.992 & -30.005 & -0.006 & & -0.034 & 30.003 & -0.007 \\
10.041 & -30.000 & -0.005 & & 5.017 & 29.997 & -0.006 \\
-20.218 & -9.995 & -0.008 & & 9.978 & 30.001 & -0.005 \\
-15.006 & -10.002 & -0.007 & & 14.996 & 30.000 & -0.007 \\
15.015 & -9.998 & -0.009 & & 19.972 & 30.002 & -0.004 \\
19.978 & -10.002 & -0.009 & & 25.031 & 29.999 & -0.003 \\
\hline
\end{tabular}

Table 2. Parameters of substitute plane.

\begin{tabular}{ccc}
\hline $\begin{array}{c}\text { Plane } \\
24 \text { points }\end{array}$ & $\begin{array}{c}\text { Parameters } \\
\text { in } \mathrm{mm}\end{array}$ & $\begin{array}{c} \pm \text { Uncertainties } \\
\text { in } \mu \mathrm{m}\end{array}$ \\
\hline$x_{A}$ & -0.00833 & 0.754 \\
$y_{A}$ & -2.08583 & 0.754 \\
$z_{A}$ & -0.00592 & 0.754 \\
$n_{x}$ & 0.0000377561 & 0.070 \\
$n_{y}$ & 0.0000019717 & 0.046 \\
$n_{z}$ & 0.9999999929 & 0.377 \\
\hline
\end{tabular}

Table 3. The two extreme points to the substitute feature.

\begin{tabular}{cccc}
\hline & $x$ & $y$ & $z$ \\
\hline$m$ & -20.218 & -9.995 & -0.0080 \\
$M$ & 25.0310 & 29.999 & -0.0030 \\
\hline
\end{tabular}

\subsection{Result of the law of propagation of uncertainty GUM}

From the expression of the flatness error given by equation (7), we estimated the variance associated to the flatness error by $V(d p)=J \Omega J^{T}$. The Jacobian matrix is given as follows:

$$
J=\left[\frac{\partial d p}{\partial n_{x}} \frac{\partial d p}{\partial n_{y}} \frac{\partial d p}{\partial n_{z}} \frac{\partial d p}{\partial x_{m}} \frac{\partial d p}{\partial y_{m}} \frac{\partial d p}{\partial z_{m}} \frac{\partial d p}{\partial x_{M}} \frac{\partial d p}{\partial y_{M}} \frac{\partial d p}{\partial z_{M}}\right]
$$

with

$$
\begin{aligned}
& \frac{\partial d p}{\partial n_{x}}=x_{m}-x_{M} ; \frac{\partial d p}{\partial n_{y}}=y_{m}-y_{M} ; \frac{\partial d p}{\partial n_{z}}=z_{m}-z_{M} \\
& \frac{\partial d p}{\partial x_{m}}=n_{x} ; \frac{\partial d p}{\partial y_{m}}=n_{y} ; \frac{\partial d p}{\partial z_{m}}=n_{z} \\
& \frac{\partial d p}{\partial x_{M}}=-n_{x} ; \frac{\partial d p}{\partial y_{M}}=-n_{y} ; \frac{\partial d p}{\partial z_{M}}=-n_{z}
\end{aligned}
$$

The two extremes points' $m$ and $M$ from the data sets along the normal direction $n \rightarrow$ are given in Table 3 .

The results obtained, by using the law of propagation of uncertainty, are given in Table 4.

The choice of the measured number points and the position of these points on the test surface greatly affect the flatness and associated uncertainty. A study, developed by
Table 4. Flatness and associated uncertainty obtained by the GUM method.

\begin{tabular}{cc}
\hline $\begin{array}{c}\text { Flatness error } \\
d p(\text { in } \mathrm{mm})\end{array}$ & $\begin{array}{c} \pm \text { Associated } \\
\text { uncertainty (in } \mathrm{mm})\end{array}$ \\
\hline 0.00678728 & 0.00485937 \\
\hline
\end{tabular}

Jalid et al. [16], was used to analyze the influence of the sample size on flatness estimation and the associated uncertainty. The uncertainty is estimated using the GUM method and the results of this study can be summarized as follows:

- If the number of points is higher, the representation of the surface is better.

- The number of points slightly increases the flatness error, which converges to a value that seems to be mostly probable.

- The number of points reduces the flatness uncertainty and therefore a reduced number of points overestimates the uncertainty, which may lead to an erroneous conformity decision.

\subsection{Simulation by Monte Carlo method}

\subsubsection{Data for the Monte Carlo simulation}

The function to simulate represents the flatness error $(d p)$ and expressed by

$$
d p=\left|\left(x_{m}-x_{M}\right) n_{x}+\left(y_{m}-y_{M}\right) n_{y}+\left(z_{m}-z_{M}\right) n_{z}\right| .
$$

This function dp, depends on random variables $\left(x_{m}, y_{m}, z_{m}, x_{M}, y_{M}, z_{M}, n_{x}, n_{y}, n_{z}\right)$. The hypothesis of a normal distribution of each variable is retained. Each distribution has $\mu$ as mean value and $\sigma=$ Expanded uncertainty $/ k$ as a standard deviation, with $k=2$.

The two extreme points, $m$ and $M$ are given in Table 3 in the previous paragraph, while the normal $n \rightarrow$ $(n x, n y, n z)^{t}$ with the respective uncertainties obtained, are given in Table 2 . 
Table 5. Results given by GUM and Monte carlo methods.

\begin{tabular}{cc}
\hline $\begin{array}{c}\text { Result of the law of propagation of } \\
\text { uncertainty }(\text { GUM }) \text { with } k=2(95 \%)\end{array}$ & $\begin{array}{c}\text { Result of Monte Carlo method } \\
\text { with } k=2(95 \%)\end{array}$ \\
\hline Flatness error $y=0.00678728 \mathrm{~mm}$ & Flatness error $=0.00678929 \mathrm{~mm}$ \\
\hline$\sigma_{\text {flatness }}=0.00242968 \mathrm{~mm}$ & $u(y)=0.00261165 \mathrm{~mm}$ \\
\hline$U=2 . \sigma_{\text {flatness }}=0.00485937 \mathrm{~mm}$ & $\begin{array}{c}y_{\text {low }}=0.00156599 \mathrm{~mm} \\
y_{\text {high }}=0.01201260 \mathrm{~mm}\end{array}$ \\
\hline
\end{tabular}

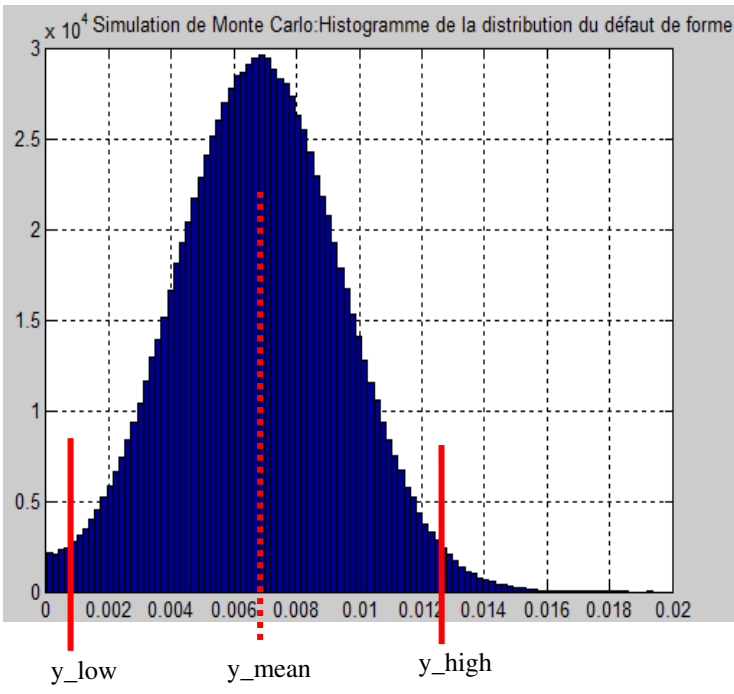

Fig. 8. Distribution of the Monte Carlo method.

\subsubsection{Generation of random variables}

To generate random variables, distributed according to a normal distribution, we used the Box Muller algorithm [17]. A program written in Matlab generates the variables $\left(x_{m}, y_{m}, z_{m}, x_{M}, y_{M}, z_{M}, n_{x}, n_{y}, n_{z}\right)$ belonging to a normal distribution of each variable. We allowed the simulation according to the Monte Carlo simulation. The obtained results are plotted in Figure 8.

We retain the following values:

- The output average value called $y_{\text {mean }}=$ $0.00678929 \mathrm{~mm}$.

- The standard deviation of the distribution $y_{\text {std }}=$ $0.00261165 \mathrm{~mm}$.

- The $y_{\text {low }}$ and $y_{\text {high }}$ values are given $95 \%(k=2)$ :

$$
\begin{aligned}
y_{\text {low }} & =0.00156599 \\
y_{\text {high }} & =0.01201260 .
\end{aligned}
$$

\subsection{Comparison of the results given by the two methods}

Bellow, we give the procedure for validation of the GUM approach, by Monte Carlo simulation (see Fig. 9).

From the data points and the coordinates uncertainties, we estimated the parameters and uncertainties of the substitute surface. The model giving the flatness deviation is used in both the GUM and Monte Carlo to determine the result and associated uncertainty. The compraison is performed to verify compliance with the criterion defined in the GUM supplement 1.

The results given by the two methods are summarized in Table 5 .

It is noted that, the estimated value of the flatness error obtained by the two methods is almost the same (difference of about $10^{-6}$ ) and the difference between the confidence intervals is very low. To confirm, the validity of the results found by the GUM uncertainty propagation, the intervals $\left|y-U(y)-y_{\text {low }}\right|$ and $t\left|y+U(y)-y_{\text {high }}\right|$ must be less than $\xi$.

The numerical tolerance is $\xi=0.5 \times 10^{-4}$ and the two intervals are calculated as follows:

$$
\begin{aligned}
\left|y-U(y)-y_{\text {low }}\right|= & \mid 0.00678728-0.00485937 \\
& -0.00156599 \mid \\
= & 0.361 \times 10^{-5}<0.5 \times 10^{-4} \\
\left|y+U(y)-y_{\text {high }}\right|= & \mid 0.00678728+0.00485937 \\
& -0.01201260 \mid \\
= & 0.365 \times 10^{-5}<0.5 \times 10^{-4} .
\end{aligned}
$$

After calculation, we find out that, the criteria described by the GUM Supplement 1, are respected in both cases. In this respect, we can conclude that the developed model, based on the law of propagation of uncertainty is valid.

\section{Conclusion}

The proposed article presents, a comparative study of the methods for estimating the uncertainty of measurement applied to a flatness error. We began by presenting the different methods of propagation of uncertainties, namely the GUM approach and Monte Carlo simulation. From a set of points belonging to a surface test on CMM, the estimation of the parameters of the substitute plane and the associated uncertainties, are performed based on the orthogonal distance regression algorithm (ODR). Moreover, we estimated the flatness error and the associated uncertainty, according to the GUM and the Monte Carlo methods. The results found by the two methods are then compared. We can retain that the flatness deviation found by the two methods is nearly identical (gap of about $10^{-6}$ ) and that the difference between the two obtained confidence intervals is very low. The criterion described by the GUM Supplement 1 is respected. Thus, we can conclude that the developed model, based on the law of propagation of uncertainty is valid. 


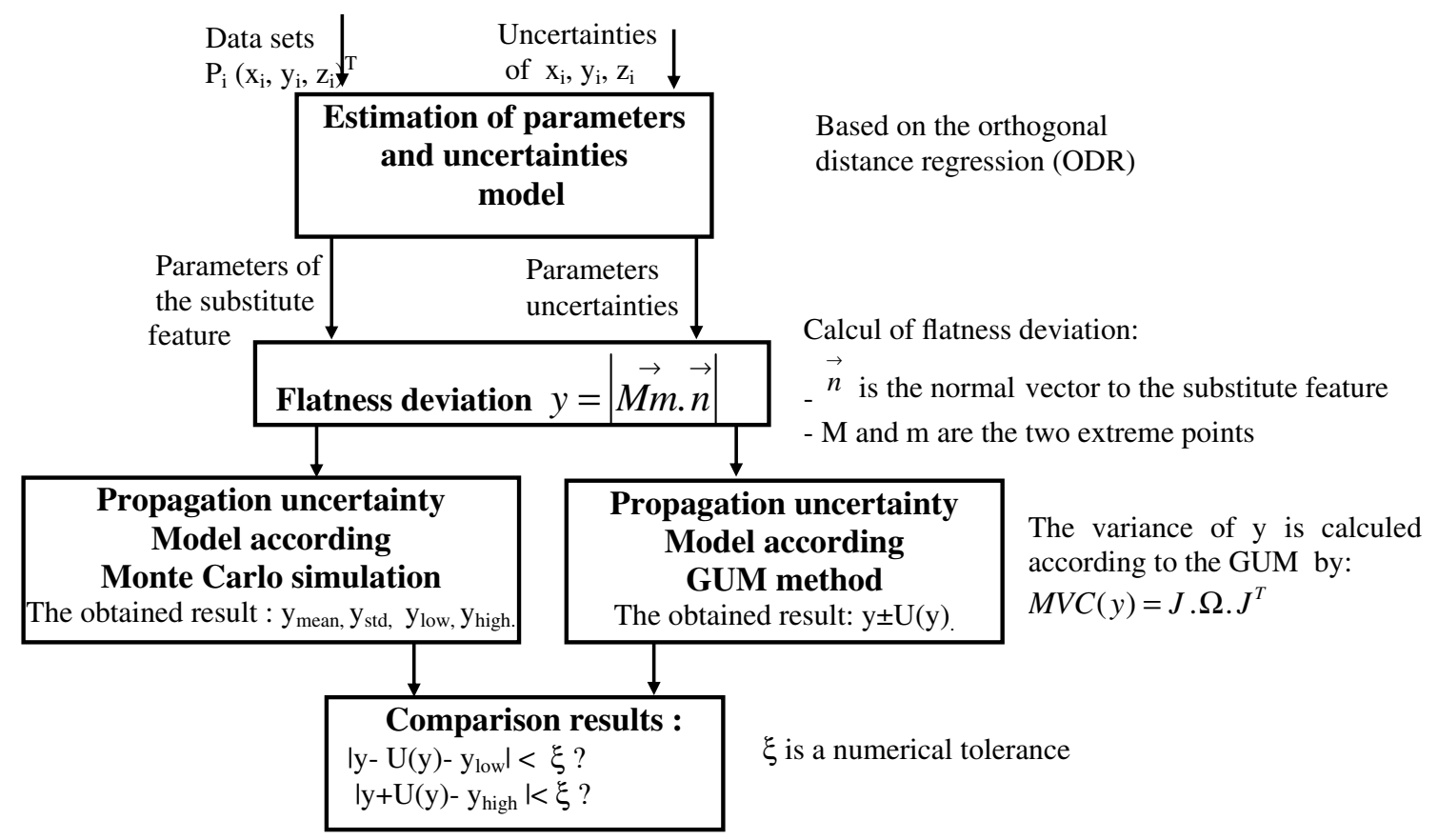

Fig. 9. Procedure for validation of the GUM approach by Monte Carlo simulation.

\section{Appendix}

The parameters of the substitute geometry are estimated by using the orthogonal distance regression (ODR) model. The solution is iteratively found using a trust region Levenberg-Marquardt method.

A method is developed in reference [14], which gives more details about the algorithm as well as the adopted approach.

ODRPACK finds the solution of an implicit orthogonal distance regression problem using the classic quadratic penalty function method. The penalty function is given as follows:

$$
\begin{aligned}
P\left(\beta, \delta ; r_{k}\right)= & \sum_{i=1}^{n}\left(r_{k}\left[f_{i}\left(x_{i}+\delta_{i} ; \beta\right)\right]^{T}\left[f_{i}\left(x_{i}+\delta_{i} ; \beta\right)\right]\right. \\
& \left.+\left[\delta_{i}^{T} \omega_{\delta_{i}} \delta_{i}\right]\right)
\end{aligned}
$$

with penalty term a

$$
\sum_{i=1}^{n} r_{k}\left[f_{i}\left(x_{i}+\delta_{i} ; \beta\right)\right]^{T}\left[f_{i}\left(x_{i}+\delta_{i} ; \beta\right)\right]
$$

and penalty parameter $r_{k}$.

A sequence of unconstrained minimization problems given as follows: $\min _{\beta, \delta} P\left(\beta, \delta ; r_{k}\right)$ is then solved for a sequence of values of the penalty parameter $r_{k}$ tending to $\infty$. The iterations are stopped when any one of the three stopping criteria are met. The weighted sum of the squared observation errors is sufficiently small, or the change in the estimated values of $\beta$ and $\delta$ is sufficiently small. The third stopping criterion is a limit on the number of iterations.

The algorithm of this method is implemented in ODRPACK, Boggs et al. [13].

\section{References}

1. J. Sladek, A. Gaska, Evaluation of coordinate measurement uncertainty with use of virtual machine model based on Monte Carlo method, Measurement 45, 1564-1575 (2012)

2. X.-L. Wen, X.-C. Zhu, Y.-B. Zhao, D.-X. Wang, F.-L. Wang, Flatness error evaluation and verification based on new generation geometrical product specification (GPS), Article, Precision Engineering 36, 70-76 (2012)

3. A.B. Forbes, Approaches to evaluating measurement uncertainty, Article, Int. J. Metrol. Qual. Eng. 3, 71-77 (2012)

4. A. Balsamo, M. Di Ciommo, R. Mugno, B.I. Rebeglia, E. Ricci, R. Grella, "Evaluation of CMM uncertainty through Monte Carlo simulations", CIRP Ann. - Manuf. Technol. 48, 425-428 (1999). Montreux, Switzerland.

5. J.-P. Kruth, N. Van Gestel, P. Bleys, F. Welkenhuyzen, Uncertainty determination for CMMs by Monte Carlo simulation integrating feature form deviations, CIRP Ann.Manufa. Technol. 58, 463-466 (2009)

6. Changcai Cui, Shiwei Fu, Fugui Huang, Research on the uncertainties from different form error evaluation methods by CMM sampling, Int. J. Adv. Manuf. Technol. 43, 136$145(2009)$

7. M.G. Cox, B.R.L. Siebert, The use of a Monte Carlo method for evaluating uncertainty and expanded uncertainty, Metrologia 43, S178 (2006).

8. J.-Z. Yang, G.-X. Li, B.-Z. Wu, J. Wang, Comparison of GUF and Monte Carlo methods to evaluate task-specific uncertainty in laser tracker measurement, J. Central South Univ. 21, 3793-3804 (2014)

9. C. Diaz, T.H. Hopp, Testing of coordinate measuring system software, in: Proceedings of 1993 American Society for Quality Control Measurement Quality Conference, 1993

10. JCGM 100:2008, Evaluation of measurement data - Guide to the expression of uncertainty in measurement, 2008 
11. JCGM 101, Evaluation of measurement data - Supplement 1 to the Guide to the expression of uncertainty in measurement - Propagation of distributions using a Monte Carlo method, BIPM Joint Committee for Guides in Metrology, Sevres, 2008

12. International Organization for Standardization ISO 1101: 2004, Geometrical product specifications (GPS) Geometrical tolerancing - Tolerances of form, orientation, location and run-out, Norme, 2004

13. P.T. Boggs, R.H. Byrd, J.E. Rogers, R.B. Schnabel, Users Reference Guide for ODRPACK version 2.01, Software for Weightes Orthogonal Distance Regression, 1992
14. A. Jalid, S. Hariri, J.P. Senelaer, Estimation of form deviation and the associated uncertainty in coordinate metrology, Int. J. Qual. Reliab. Manage. 32 (2015)

15. International Standard Development of Virtual CMM, Final Research Report, the University of Tokyo, Japan, May 2002, p. 72

16. A. Jalid, S. Hariri, N.E. Laghzale, Influence of sample size on flatness estimation and uncertainty in threedimensional measurement, Int. J. Metrol. Qual. Eng. 6, $101(2015)$

17. G.E.P. Box, M.E. Muller, A note on the generation of random normal deviates, Ann. Math. Stat. 29, 610-611 (1958) 